\title{
Corpus
}

\section{Prenons nos distances pour comparer des textes, les analyser et les représenter}

Jean-Pierre Barthélémy, Xuan Luong et Sylvie Mellet

\section{(2) OpenEdition}

1 Journals

\section{Édition électronique}

URL : http://journals.openedition.org/corpus/25

DOI : $10.4000 /$ corpus. 25

ISSN : $1765-3126$

\section{Éditeur}

Bases; corpus et langage - UMR 6039

\section{Édition imprimée}

Date de publication : 15 décembre 2003

ISSN : 1638-9808

\section{Référence électronique}

Jean-Pierre Barthélémy, Xuan Luong et Sylvie Mellet, « Prenons nos distances pour comparer des textes, les analyser et les représenter », Corpus [En ligne], 2 | 2003, mis en ligne le 15 décembre 2004, consulté le 08 septembre 2020. URL : http://journals.openedition.org/corpus/25 ; DOI : https:// doi.org/10.4000/corpus. 25

Ce document a été généré automatiquement le 8 septembre 2020

(c) Tous droits réservés 


\title{
Prenons nos distances pour comparer des textes, les analyser et les représenter
}

\author{
Jean-Pierre Barthélémy, Xuan Luong et Sylvie Mellet
}

\section{Prolégomènes}

1 L'usage de méthodes mathématiques pour traiter de données textuelles a une longue tradition. On se souvient que c'est en étudiant Pouchkine que Markov a élaboré ses chaînes. La linguistique mathématique (qui dépasse de très loin l'analyse des données textuelles) a connu un peu partout un essor important et a fourni des modèles à bon nombre de travaux portant sur le langage (Chomsky, Harris, Montague, ...). On connaît aussi, via des mathématiques fort sophistiquées, la profonde influence réciproque entre la linguistique formelle et l'informatique (théorie des automates, théorème de ChurchRosser, lamda calcul...). Mais revenons au sujet de la présente livraison de CORPUS : les distances textuelles, qui permettent d'évaluer et de représenter la proximité ou l'éloignement entre deux ou plusieurs textes en fonction de divers paramètres (dont le plus familier et le plus fréquemment étudié est leur lexique). Les mathématiques sont ici beaucoup plus simples; elles font appel à quelques rudiments sur les «espaces métriques discrets " souvent couplés avec des méthodes de représentation: calcul statistique (articles de C. Labbé \& D. Labbé, de T. Merriam), analyses factorielles (articles de M. Bécue-Bertaut, d'É. Brunet, de M. Kastberg Sjöblom, de D. Longrée \& X. Luong, de D. Valentin et al. ), multidimensional scaling (MDS ${ }^{1}$, article de T. Merriam) et représentation arborée (article de X. Luong \& S. Mellet, et aussi d'É. Brunet, de M. Kastberg Sjöblom). Par espace métrique discret nous entendons que des textes sont comparés deux à deux à l'aide d'une distance (ou d'une mesure de similitude, ou d'une mesure de dissimilarité). Celle-ci peut être plus ou moins dépendante de la méthode de représentation utilisée en aval. Par exemple les analyses de correspondances marquent une nette prédilection pour la distance du khi-2. Toutes les mesures de dissimilarité (ou presque) peuvent se prêter à une représentation arborée ou à une représentation issue du MDS. Ces représentations ne sont cependant pas égales relativement à la fiabilité des interprétations. Les arbres aiment bien s'appuyer sur des distances de Manhattan ${ }^{2}$ ou 
issues du modèle du contraste de Tverky (1977) ; la violation de l'inégalité triangulaire crée de méchants phénomènes d'hyperbolicité ${ }^{3}$ en MDS.

\section{Précautions d'emploi}

Les analystes "statisticiens» de données textuelles marchent sur le fil d'un rasoir. Cette déambulation leur fait encourir, au moins, trois risques :

- i. se couper la plante des pieds (même si couper les plantes du pied d'un arbre peut lui redonner force et vigueur) ;

- ii. choir d'un côté ;

- iii. choir de l'autre.

(i) Le parti pris est d'adapter des textes aux instruments numériques qui seront mis en œuvre pour les comparer. Classiquement, un corpus est réduit à des formes lexicales, lemmatisées ou non, selon un procédé puisé au sein d'une panoplie de pratiques possibles. On peut, ainsi, ne s'intéresser qu'à l'usage des "mots ", des substantifs, de l'environnement d'un thème générique (l'amour, la guerre, ...), des phonèmes, des signes de ponctuation, de certaines catégories grammaticales, ... Il n'y a ici que des approches locales, fortement pilotées par des a priori de recherche particuliers. Les linguistes, les philologues, les spécialistes de la littérature n-ièmiste suivent des pistes qui, tout en s'affirmant plus globales, sont aussi fondées sur d'autres érudites localités.

(ii) En fonction de l'usage qui en est fait, toute métrique présente une part d'arbitraire et des biais. Lorsqu'elle est libre relativement à une méthode de représentation, elle peut avoir une grande influence sur les résultats. Une méthode peut aussi se révéler pertinente pour la comparaison de textes et mal se prêter à un type de représentation donné. Par ailleurs, lorsque les sorties (représentations) forcent peu ou prou les entrées (métriques), ces dernières peuvent s'avérer peu fiables pour comparer, en amont, des textes.

5 (iii) Seules les statistiques descriptives et exploratoires, et non pas inférentielles et confirmatoires, sont sollicitées ici. Elles sont soumises à un certain nombre de contraintes qui limitent pour une part leur puissance heuristique. Compte tenu des simplifications opérées sur le lexique, on passe des signes aux formes; on ne traite qu'une partie des informations textuelles sélectionnée de manière relativement arbitraire. Le choix d'une distance est lui-même en partie arbitraire (cf. ii). Enfin le choix d'une représentation peut poser d'épineux problèmes. Ainsi dans les méthodes factorielles on retrouve exactement (sous une présentation plus intelligible) ce que procure une distance de départ, mais à condition d'en explorer toutes les dimensions.

6 Cependant deux écueils sont sur la route de l'analyste. Le premier, assez bien contourné (encore que...), est l'écart entre le modèle calculé et les données observées. Celui-ci peut être évalué de diverses manières (écarts-types, contributions, corrélations, "stress » pour le MDS, et d'autres outils plus élaborés dans le cas des analyses arborées). Un autre arbitraire se glisse ici : telle ou telle méthode apparaîtra comme plus ou moins performante selon telle ou telle évaluation de l'approximation. Un second écueil, encore plus redoutable, concerne la structure algorithmique des approches. Prenons l'exemple des représentations arborées utilisées ici par É. Brunet , M. Kastberg Sjöblom, X. Luong \& S. Mellet. Elles correspondent à un problème, qualifié dans le jargon de l'algorithmique de NP-difficile. Cela veut dire que s'il y a, sans conteste, une solution exacte, la fin du monde sera arrivée avant qu'un ordinateur en soit venu à bout (sauf si les machines programmables ont vocation à prendre le relais du monde physique). Même si on peut évaluer l'adéquation d'un arbre aux données 
textuelles qu'il est censé représenter, rien ne garantit que l'algorithme qui l'a trouvé a fourni le meilleur possible.

$7 \quad$ Les trois points soulignés ci-dessus nous semblent essentiels. La vocation des méthodes de "statistique descriptive» en analyse de données textuelles n'est pas de trancher entre des hypothèses, mais d'en suggérer. (Nous les aimons lorsqu'elle sont contreintuitives). Cependant, compte tenu des réserves présentées en (i), (ii) et (iii), le calculateur ne saurait se substituer au linguiste. Quant à la validation d'une hypothèse (accompagnée d'une probabilité d'erreur) elle relève, pour sa part, de la "statistique inférentielle». Celle-ci repose aussi sur des réductionnismes, sans doute aussi prégnants que ceux que l'on rencontre dans les articles de cette livraison, mais qui se nichent dans le choix de modèles probabilistes, évités par les approches descriptives.

3. Métriques intra et inter-textuelles

8 Nous avons déjà beaucoup parlé de distances, de mesures de similitude et de dissimilarité. De quoi s'agit-il exactement?

9 Une dissimilarité sur un ensemble fini $\mathrm{X}$ est tout simplement une application définie sur le produit cartésien $\mathrm{X}$ [F] $\mathrm{X}$, qui à tout couple $(\mathrm{x}, \mathrm{y})$ associe un nombre réel $\mathrm{d}(\mathrm{x}, \mathrm{y})$ tel que

$\mathrm{d}(\mathrm{x}, \mathrm{y}) \geq 0$,

$d(x, y)=d(y, x)$

$d(x, x)=0$

10 On a coutume de distinguer les dissimilarités propres qui vérifie $\mathrm{d}(\mathrm{x}, \mathrm{y})=0$ lorsque $\mathrm{x}=\mathrm{y}$. Techniquement, on appelle distance une dissimilarité propre qui vérifie une inégalité dite triangulaire

$$
\mathrm{d}(\mathrm{x}, \mathrm{y}) \leq \mathrm{d}(\mathrm{x}, \mathrm{z})+\mathrm{d}(\mathrm{z}, \mathrm{y}) \text { pour tout } \mathrm{x}, \mathrm{y}, \mathrm{z}
$$

11 Une mesure de similitude varie en sens inverse d'une dissimilarité. Elle prend ses valeurs maximales sur les couples de la forme $(\mathrm{x}, \mathrm{x})$.

12 En analyse de données textuelles on dispose d'abord d'un corpus. Suivant l'objectif poursuivi celui-ci peut être un seul texte, divers textes d'un même auteur (article de M. Kastberg Sjöblom), divers textes d'une même époque (article de X. Luong \& S. Mellet), divers textes correspondant à un même genre (article de T. Merriam), ou à des genres différents. Ces textes ne sont pas forcément "littéraires", ils peuvent être issus d'enquêtes (article de M. Bécue-Bertaut), de comptes rendus, de recueils provenant d'expérimentation, par exemple en psychologie (article de D. Valentin et al.). Nous avons vu que des formes, qui correspondent à l'objet de l'étude, en étaient extraites. Elles constituent les lexiques sur la base desquels les textes seront comparés par le biais de mesures de dissimilarité et/ou de similitude. On trouvera dans l'article d'é. Brunet une étude comparative de ces mesures et dans l'article de C. Labbé \& D. Labbé l'étude approfondie de l'une d'entre elles.

On peut caractériser les mesures selon diverses dimensions.

- i. D'abord leur origine.

Certaines viennent, telle la "distance de Jaccard", de l'écologie. D'autres, e.g. la distance de Hamming, de la théorie de l'information. D'autres encore de la tradition mathématico-physique: distance euclidienne, ou plus généralement de Minskovski, sans compter les très nombreuses dissimilarités étudiées dans le cadre des espaces métriques discrets (Van Cutsem, 1994). D'autres ne sont pas d'importation, mais ont été 
élaborées spécifiquement pour comparer des lexiques (connexion lexicale de Muller (1964), distance de Labbé \& Labbé (2001)).

- ii. Ensuite leur caractère local ou global.

Dans le premier cas les textes sont comparés deux à deux indépendamment du corpus étudié (c'est le cas des distances de Jaccard, Hamming ...) ; dans le second les calculs sont relativisés à l'ensemble du corpus (c'est le cas de la distance du khi-2 utilisée en AFC).

• iii. Enfin, elles peuvent être de nature combinatoire ou probabiliste.

Dans le premier cas, elles peuvent être ensemblistes et sont alors fondées sur des comparaisons d'occurrences (éventuellement pondérées, en statistique on parle alors de fréquences) entre deux lexiques L et L'. Se trouvent ainsi mobilisées les formes communes à L et L', celles qui sont dans L et non pas dans L' (ou bien dans L' et non pas dans L), ou encore celles qui sont dans L et/ou dans L'. La distance de Jaccard est un archétype de cette famille dont le modèle du contraste, développé par Tversky (1977), rend partiellement compte. Elles peuvent aussi être ordinales dans divers sens. Par exemple Degenne (1972) compare diverses annonces matrimoniales en utilisant l'ordre d'utilisation de descripteurs (âge, poids, couleur des cheveux, niveau d'étude....); on trouvera un autre exemple dans ce numéro où les ordres comparent les fréquences d'apparition de formes dans des lexiques (cf. X. Luong \& S. Mellet). Dans le second cas (distances de nature probabiliste), les mesures sont fondées en particulier (mais pas exclusivement) sur la notion d'indépendance des occurrences (cf. par exemple l'approche binômiale de Muller (1968) utilisée par Brunet (1978), (1988)).

4. Quelques bribes bibliographiques

17 Nous espérons que le thème couvert par ce numéro de CORPUS convaincra les lecteurs de la pertinence de l'usage de métriques en analyse de données textuelles. Malgré les nécessaires précautions d'emploi que nous avons mentionnées plus haut, celles-ci offrent une alternative, non pas prometteuse, mais ayant déjà tenu maintes promesses, à l'impérialisme des corrélations. On verra aussi que les applications ont des objectifs assez variés : par delà le problème de l'authentification d'un texte et de son attribution à un auteur, qui a parfois monopolisé l'attention de manière excessivement médiatique, les études linguistiques et littéraires peuvent se prévaloir de résultats très satisfaisants en matière de classification générique, les calculs de distance permettant de regrouper puis de caractériser des textes relevant d'un même sous-genre tel que le roman policier au sein du roman des $19^{\text {ème }}$ et $20^{\text {ème }}$ siècles ou tel que le récit biographique au sein de l'histoire dans l'antiquité latine. Les classifications génériques et chronologiques permettent aussi de mieux appréhender l'évolution des œuvres de certains écrivains particulièrement productifs et d'en structurer la diversité. Dans un autre type d'application, les mesures de similarités thématiques, éventuellement enrichies par la prise en compte de divers paramètres énonciatifs, sont à la base des outils actuellement les plus performants de classification et d'adressage automatique de documents (que ce soit à des fins d'archivage ou de distribution de l'information). Les calculs de distance sont aussi particulièrement performants en matière d'analyse du discours politique, comme l'ont montré entre autres les travaux de D. Labbé, de P. Lafon, de D. Mayaffre ou d'A. Salem ${ }^{4}$. Enfin, nous avons tenu à ce que d'autres disciplines des sciences humaines et sociales soient représentées dans ce rapide panorama: il s'agit ici de la sociologie et de la psychologie qui élargissent ainsi le champ d'application des méthodes exposées et en dévoilent d'autres facettes. 

d'être exhaustive, concerne plus particulièrement les thèmes des articles présentés dans cette livraison de CORPUS.

\section{BIBLIOGRAPHIE}

\section{4-1 Ouvrages généraux4-1-1 Dissimilarités}

D'abord un ouvrage qui traite des propriétés mathématiques des dissimilarités :

Van Cutsem B. (1994) Classification and Dissimilarity Analysis Lecture Notes in Statistic. NewYork : Springer Verlag.

Un autre, fort utilisé en analyse de données textuelles :

Chandon J.L. \& Pinson S. (1981) Analyse Typologique. Théories et Applications. Paris : Masson.

4-1-2 Analyse factorielle

Citons deux ouvrages de base pour l'analyse factorielle des correspondances, et la distance du khi-2 appliquées aux textes :

Benzécri J.P. et alii (1981) Linguistique et Lexicologie. Pratique de l'Analyse des Données 3. Paris : Dunod.

Lebart L. \& Salem A. (1994) Statistique textuelle. Paris : Dunod.

4-1-3 Multidimensional scaling

Torgerson W.S. (1958) Theory and methods of Scaling. New-York : Wiley.

Shepard R.N. et alii (eds) (1972) Multidimensional Scaling : Theory and applications in the behavioral Sciences. Vol. 1 : Theory. New York : Seminar Press.

Kruskal J.B. \& Wish M. (1978) Multidimensional Scaling. Beverly Hills \& London : Sage.

4-1-4 Techniques ordinales

Degenne A. (1972) Techniques ordinales en analyse des données. Paris : Hachette.

Barthélémy J.P. (1979) Propriétés métriques des ensembles ordonnés. Comparaison et agrégation des relations binaires. Thèse de doctorat d'Etat : Besançon.

Abdi H. (1981) Approches préordinales et Analyse de Données psychologique. Thèse : Université d'Aix-en-Provence.

4-1-5 Analyse arborée

Luong X. (éd) (1989) Analyse arborée des données textuelles - Tree analysis of textual data. CUMFID n¹6, Nice : Institut National de la Langue Française; CNRS ; 178 p.

Barthémemy J.P. \& Guénoche A. (1991) Trees and Proximity Representation. New-York : Wiley \& son.

4-2 Connexion lexicale

C. Muller a produit une œuvre de pionner en introduisant la notion de connexion lexicale. Celleci se laisse construire de deux manières, l'une ensembliste et l'autre qui s'appuie sur la loi 
binômiale pour tenir compte des fréquences. É. Brunet, du temps héroïque des cartes perforées, l'a élaborée et instanciée sur de vastes ensembles de données.

Muller C. (1964) Etude de statistique lexicale. Le vocabulaire de Pierre Corneille. Paris : Larousse.

Muller C. (1968) Initiation à la statistique linguistique. Paris : Larousse.

Brunet É. (1978) Le vocabulaire de Jean Giraudoux. Structure et évolution. Troisième partie, Chapitre I (pp. 369-396) : La connexion lexicale. Genève : Slatkine.

Brunet É. (1988) Le vocabulaire de Victor Hugo. Quatrième partie, Chapitre I (pp. 275-299) : La connexion lexicale. Genève / Paris : Slatkine - Champion.

Représentations arborées des données de Brunet (connexion lexicale, version probabiliste) :

Barthélemy J.P. \& Luong X. (1987) «Sur la topologie d'un arbre phylogénétique : aspects théoriques, algorithmiques et applications à l'analyse de données textuelles », Math. et Sciences Humaines $\mathrm{n}^{\circ} 100$, pp. 57-80.

4-3 Analyse en composantes principales

Voici quelques références sur les analyses de tableaux lexicaux à l'aide de distances euclidienne et de l'analyse en composantes principales :

Burrows J.F. (1989) « An Ocean Where Each Kind... : Statistical Analysis and Some Major Determinants of Literary Style », Computers and the Humanities n²3, pp. 309-321.

Tabata T. (1994) « Dickens Narrative Style : A Statistical Approach to Chronological Variation », RISSH n³0, pp. 165-182.

Holmes D. I. \& Forsyth R.S. (1995) « The Federalist Revisited : New Directions in Authorship Attribution », Literary and Linguistic Computing n ${ }^{\circ} 10$, pp. 111-127.

Baayen H., van Halteren H. \& Tweedie F. (1996) « Outside the Cave of Shadowss: Using Syntactic Annotation to Enhance Authorship Attribution», Literary and Linguistic Computing n ${ }^{\circ} 11, \mathrm{pp}$.

121-131.

Tweedie F., Holmes D. \& Corns T. (1998) « The Provenance of De Doctrina Christiana, Attributed to John Milton : A Statistical Investigation », Literary and Linguistic Computing n ${ }^{\circ} 13$, pp. 77-88.

Craig H. (1999) « Authorial Attribution and Computationnal Stylistic », Literary and Linguistic Computing $\mathrm{n}^{\circ} 14$, pp. 103-113.

Binongo J.N.G. \& Smith M.W.A. (1999) « The Application of Principal Component Analysis to Stylometry ", Literary and Linguistic Computing n¹4, pp. 445-465.

Burrows J.F. \& Love H. (1999) « Attribution Tests and The Editing of Seventeenth-century Poetry », Yearbook of English Studies n²9, pp. 151-175.

4-4 Multidimensional Scaling

Quelques références relatives à des pratiques euclidiennes : distances euclidiennes, dissimilarités construites à partir des coefficients de corrélation et représentations issues du MDS :

Woelfel J. \& Fink E.L. (1980) The Measurement of Communi-cation Processes : Galileo Theory and Method. New-York : Wiley.

Burrows J.F. (1987) Computation into Criticism : A Study of Jane Austen's Novels and an Experiment in Method. Oxford: Oxford University Press.

Potter R.G. (1991) « Statistical Analysis of Literature : A Retrospective on Computers and the Humanities, 1966-1990 », Computers and the Humanities n²5, pp. 271-293. 
Horton T.B. (1991) « Frequent Words, Authorship, and Characterization in Jacobean Drama » in Hockey \& Ide (eds), Research in Humanities Computing. Oxford : Oxford University Press, pp. 47-69.

Holmes D. I. (1994) « Authorship attribution », Computers and the Humanities n²8, pp. 1-20.

Burrows J.F. \& Craig H. (2001) « Lucy Hutchinson and the Authorship of Two Sevententh-century Poems : A computationnal Approach », The Sevententh Century n¹6, pp. 259-282.

Spencer M. et alii (2003) « Analysing the Order of Items in Manuscripts of The Canterbury Tales », Computers and the Humanities n³7, pp. 97-109.

\section{4-5 Représentations arborées4-5-1 Théories et Méthodes}

Tversky A. (1977) « Features of Similarity », Psychological Review n²4, pp. 327-352.

Abdi H., Barthélemy J.P. \& Luong X. (1988) « Représentations arborées et catégories naturelles » in Analyse Arborée des données textuelles, CUMFID n¹6, pp. 156-179.

Juillard M. \& Luong X. (1989) « Unrooted Tree Revisited: Topology and Poetic Data », Computers and the Humanities $n^{\circ} 23$, pp. 215-225.

Luong X. (1994) « Analyse arborée des données textuelles, mode d' emploi », Travaux du Cercle Linguistique $\mathrm{n}^{\circ} 16$, Université de Nice, pp. 25-42.

Barthélemy J.P. \& Luong X. (1998) « Représenter les données textuelles par les arbres... », in S. Mellet (éd) JADT 1998, 4èmes Journées Internationales d'Analyse Statistique des Données Textuelles. Nice, pp. 49-71.

\section{4-5-2 Applications}

Juillard M. \& Luong X. (1988) « As the leaves grow on the tree : a new way to analyze linguistic data ", Literary and Linguistic Computing, vol. 3, n², pp. 125-131.

Juillard M. \& Luong X. (1994) « De nouveaux arbres pour un nouveau corpus », Revue Informatique et Statistique dans les Sciences humaines, vol. 24, n¹-4, pp. 221-240.

Juillard M. \& Luong X. (1997) « Words in the hood : a new look at the distribution of words in text », Linguistic and Computing, pp. 47-65.

Luong X. \& Maciel C. (1999) « Le vocabulaire des constitutions des pays lusophones. Essai d'application d'un modèle mathématique » in Os desafios da lusophonia, Hommage à R.A. Lawton. CUMFID ${ }^{\circ}{ }^{19}$. Nice.

Dubrocard M. \& Luong X. (2000) «Problèmes d'attribution : application de quelques tests statistiques à différents historiens latins, analyse arborée » Actes du Colloque Internatinal VEXTAL.Venise.

Cottier J.F., Dubrocard M. \& Luong X. (2002) « Détermination de quelques manuscrits du latin médiéval », Médiévales n42, pp. 55-71.

Luong X. \& Mayaffre D. (2004) « Arbres et généalogie politique. Représentation arborée du discours de Jacques Chirac (1995-2002) », Histoire et Mesure, sous presse.

4-6 Constructions de mesures de (dis)similarité ou de distance diverses

Brunet É. \& Muller C. (1988) «La statistique résout-elle les problèmes d'attribution? », Strumenti critici, III, $\mathrm{n}^{\circ} 3$, pp. 367-387.

Luong X. \& Mellet S. (1995) « Les calculs multidimensionnels au service de l'analyse syntaxique diachronique » in Analisi Statistica dei Dati Testuali. Roma : CISU, pp. 281-288. 
Évrard É. (1996) «Étude des dialectes bantous » in Statistique et analyse linguistique. Paris : PUF, pp. 85-103.

Hubert P. \& Labbé D. (1998) « La connexion des vocabulaires » in S. Mellet (éd) JADT 1998, $4^{\text {èmes }}$ Journées Internationales d'Analyse Statistique des Données Textuelles. Nice, pp. 361-369.

Brunet É. (1999) «Ce que disent les chiffres », in Nouvelle Histoire de la langue française, Paris : Éd. du Seuil, pp. 673-727.

Labbé D. \& Monière D. (2000) « La connexion intertextuelle. Application au discours gouvernemental québécois ", in Rajman \& Chappelier (eds) Actes des $5^{\text {èmes }}$ Journées Internationales d'Analyse Statistique des Données Textuelles. Lausanne, vol. 1, pp. 85-94.

Labbé C. \& Labbé D. (2001) « Textual Distance and Authorship Attribution Corneille and Molière ", Journal of Quantitative Linguistics $n^{\circ} 8,3$, pp. 213-231.

Pincemin B. (2002) «Similarités texte-textes. Expérience d'une application de diffusion ciblée et proposition ", in Matemáticas y Tratamiento de Corpus, Séminaire interlatin de linguistique appliquée, Logroño : Fundacion San Milán de la Cogolla, pp. 35-52.

Longrée D. \& Luong X. (2003) « Spécificités stylistiques et distributions temporelles chez les historiens latins : sur les méthodes d'analyse quantitative d'un corpus lemmatisé » in Actes des 2èmes Journées de la Linguistique de Corpus. Lorient (sous presse).

Brunet É. (2002) « Le lemme comme on l'aime », in A. Morin et P. Sébillot (éds.) JADT 2002, $6^{\text {èmes }}$ Journées inter-nationales d'Analyse statistique des Données Textuelles. Saint-Malo : IRISA - INRIA, vol. 2, pp. 221-232.

Labbé D. \& Monière D. (2003) Le discours gouvernemental. Canada, Québec, France 1945-2000. Paris : Honoré Champion (Lettres numériques 4).

Burrows J. (2003) « Questions of Authorship: Attribution and Beyond », Computers and the Humanities $n^{\circ} 37,1$, pp. 5-32.

Somers H. \& Tweedie F. (2003) « Authorship Attribution and Pastiche», Computers and the Humanities $\mathrm{n}^{\circ} 37,2$, pp. 407-429.

\section{NOTES}

1.. Le propos du MDS est de représenter un ensemble fini de points muni d'une distance - ou d'une dissimilarité - dans un espace euclidien de manière à optimiser un certain critère (traduisant le niveau de fidélité de la représentation).

2. Cette distance, qualifiée « de type $\mathrm{L}^{1}$ » en mathématique, traduit le fait que pour aller d'un point à un autre on n'imite pas le vol d'un oiseau (distance euclidienne) mais que l'on suit des rues qui se coupent à l'angle droit comme dans la partie de Manhattan située avant Greenwich village. On l'appelle aussi « city block distance ».

3.. Il s'agit là d'un point très technique. Lorsqu'une dissimilarité ne vérifie pas l'inégalité triangulaire (cf. infra p. 9) sa représentation dans un espace euclidien est fortement biaisée par d'inexorables courbures.

4.. Cf., entre autres, D. Labbé (1983) François Mitterand : essai sur le discours, Grenoble : La Pensée sauvage ; P. Lafon (1984) Dépouil-lements et statistiques en lexicométrie, Paris : Champion ; D. Mayaffre (2000) Le poids des mots. Le discours de gauche et de droite dans l'entre-deux-guerres, Paris : Champion; A. Salem (1987) Pratique des segments répétés, Essai 
de statistique textuelle, Paris : Klincksieck; A. Salem (1988) « Approches du temps lexical », Mots n¹7, pp. 105-143; et de nombreux numéros de la revue Mots.

\section{AUTEURS}

JEAN-PIERRE BARTHÉLÉMY

L.I.A.S.C., ENST de Bretagne

XUAN LUONG

"Bases, Corpus et Langage", UMR 6039, Nice

SYLVIE MELLET

"Bases, Corpus et Langage", UMR 6039, Nice 